\title{
Symptom burden and integrative medicine in cancer survivorship
}

\author{
Susanne M. Cutshall • Stephen S. Cha • Sheryl M. Ness • \\ Daniela L. Stan • Sarah A. Christensen • Anjali Bhagra • \\ Katharine A. Price • Carrie A. Thompson • \\ Shahrukh K. Hashmi • Tony Y. Chon • Tracy J. McCray • \\ Amit Sood • Brent A. Bauer • Kathryn J. Ruddy
}

Received: 14 October 2014 / Accepted: 15 February 2015 /Published online: 28 February 2015

(C) Springer-Verlag Berlin Heidelberg 2015

\begin{abstract}
Purpose The purpose of the study was to learn about the level of experience with, interest in, and desire for knowledge about integrative medicine (IM) among cancer survivors.

Methods Cancer survivors attending the 2014 National Cancer Survivors Day in Rochester, MN, were recruited to participate in a one-page survey about their ongoing health concerns and symptoms, as well as their experience with, interest in, and desire
\end{abstract}

Statement of relevance It is critical that oncology providers help cancer survivors address ongoing health concerns. This article provides background information on common health concerns of cancer survivors and use of integrative medicine techniques to address them. Education about and access to evidence-based integrative medicine techniques may have important roles in comprehensive cancer survivorship programs.

S. M. Cutshall

Department of General Internal Medicine, Mayo Clinic,

Rochester, MN, USA

\section{S. S. Cha}

Division of Biomedical Statistics and Informatics, Mayo Clinic, Rochester, MN, USA

S. M. Ness $\cdot$ S. A. Christensen

Cancer Center, Mayo Clinic, Rochester, MN, USA

D. L. Stan • A. Bhagra · T. Y. Chon • A. Sood • B. A. Bauer Division of General Internal Medicine, Mayo Clinic, Rochester, MN, USA

\section{K. A. Price $\cdot$ K. J. Ruddy $(\square)$}

Division of Medical Oncology, Mayo Clinic, 200 First St SW,

Rochester, MN 55905, USA

e-mail: ruddy.kathryn@mayo.edu

C. A. Thompson • S. K. Hashmi

Division of Hematology, Mayo Clinic, Rochester, MN, USA

T. J. McCray

Public Affairs, Mayo Clinic, Rochester, MN, USA for knowledge about IM. Two-sided $t$ test was used for univariate analyses of impact of sex, time since diagnosis, and age.

Results Among the 260 cancer survivors, 171 persons (female, $74 \%$; male, $26 \%$ ) completed the survey (mean age, 64.6 years). Symptoms most commonly somewhat or more bothersome were fear of recurrence $(52 \%)$, stress (43\%), fatigue (43\%), difficulty sleeping (33\%), and weight gain (31\%). The most used IM resources were exercise (75\%), improved nutrition and diet (66\%), stress management (42\%), dietary supplementation (33\%), meditation ( $25 \%$ ), and massage ( $22 \%$ ). Older patients (age, $\geq 65$ years) were less experienced with, interested in, and desiring of knowledge about IM techniques. Sex and time since diagnosis were not strongly predictive of most survey response categories.

Conclusions Cancer survivors have adverse effects for years into survivorship. They use and express interest in various IM techniques to help manage symptoms. It is critical that oncology providers help survivors address ongoing health concerns. Education about and access to evidence-based IM techniques may have important roles in comprehensive cancer survivorship programs.

Keywords Alternative medicine $\cdot$ Cancer . Cancer symptoms $\cdot$ Complementary medicine . Integrative medicine $\cdot$ Survivorship

\section{Abbreviation \\ IM Integrative medicine}

\section{Introduction}

In the USA, the number of cancer survivors has more than tripled over the past 30 years; nearly 14 million persons live with a history of cancer. This number is expected to grow to 
18 million by 2018 . As the number of survivors dramatically increases, in part because of improved treatments, reliable information is needed about the posttreatment cancer experience. Some cancer survivors use integrative medicine (IM) therapies (e.g., acupuncture, massage, meditation) to help manage the emotional and physical sequelae of cancer. To comprehensively assess the current health concerns of cancer survivors and to assess the use of and interest in IM in this population, we conducted a survey of cancer survivors who attended the annual National Cancer Survivors Day event in Rochester, MN, in 2014.

\section{Cancer survivorship concerns}

Cancer survivors face many late and long-term treatment toxicities, including pain [1, 2], fatigue [2, 3], neuropathy [2, 4], lymphedema [5], heart disease [6], difficulty sleeping [2, 7], weight gain [8], cognitive dysfunction [9-11], and sexual dysfunction [12-14]. They often experience social and emotional sequelae of cancer and its treatment, including fear of recurrence [7, 15], stress [7, 16], and financial issues [17]. Research is ongoing to assess how best to address these concerns.

\section{Mayo Clinic cancer survivorship care}

Coordination and optimization of cancer survivorship services are a major institutional priority at Mayo Clinic. Mayo sees 13,000 new cancer patients annually across its 3 primary sites in Rochester, MN; Jacksonville, FL; and Scottsdale, AZ, as well as at affiliated facilities. Eight disease-specific survivorship clinics operate within the Mayo Clinic enterprise, and new survivorship clinics are in development. Visits to a Mayo survivorship clinic typically include a review of cancer history and physical examination, an assessment of physical and psychosocial effects of cancer and its treatment, recommendations regarding lifestyle, provision of a treatment summary and survivorship care plan, and referrals as needed (e.g., to physical therapy, psychology, cardio-oncology, and IM). However, only a small fraction of Mayo Clinic cancer patients (approximately 1000 annually) are served by its existing survivorship clinics, and little is known about the unmet needs in this population, particularly in those with non-breast cancers because to date, most survivorship research has focused on breast cancer. Additional research is needed to assess symptom burden and potential supportive interventions, and it is important that male patients and patients who have rarer cancers be studied.

Integrative therapies for cancer survivors

IM is a health care specialty that focuses on the holistic needs of the entire person (i.e., physical, emotional, and spiritual). Techniques such as massage, acupuncture, and meditation are used to improve a person's overall feeling of health and wellness, often with less emphasis on treating a specific disease than there is in traditional medicine.

Interest is growing in the concomitant use of IM and conventional medicine in the care of cancer survivors. For example, some cancer survivors find that acupuncture is helpful for management of late and long-term treatment adverse effects. One small, randomized controlled trial $(N=80)$ suggested that acupuncture was more beneficial than fluoxetine for sleep [18], and four randomized controlled trials (patient numbers ranged from 50 to 302) suggested that acupuncture reduced depression more than medications or usual conventional medical care [18-22]. Acupuncture also was found to be a better therapy for anxiety than usual care $(N=302)$ [22]. However, data are limited and mixed (particularly from studies that randomly assigned participants to real or sham acupuncture) $[23$, 24].

Stress reduction and resilience training based in mindfulness have shown promising results. A small single-blind trial $(N=25)$ randomly assigned breast cancer survivors to two 90 minute group training sessions, a brief individual session, and three follow-up telephone calls over 12 weeks vs a wait-list control [25]. The study investigators found improvements in resilience, perceived stress, anxiety, and overall quality of life at 12 weeks. A larger $(N=271)$, multisite randomized intervention study of 18 hours of mindfulness meditation and gentle yoga (vs 18 hours of expression and group support or a 1-day stress management control group) found that the mindfulness intervention improved stress symptoms the most. Furthermore, other studies have suggested that similar interventions are promising for sleep improvement [26-28].

Little is known about the frequency of use of IM approaches in cancer survivors in the Midwestern USA or about what proportion of survivors desire more information about various IM therapies. We aimed to study these issues and to better understand the burden of symptoms and social and emotional consequences in cancer survivors attending a National Cancer Survivors Day in Rochester, MN.

\section{Materials and methods}

Participants and procedures

This survey research protocol was approved by the Mayo Clinic Institutional Review Board. Participants were recruited at the National Cancer Survivors Day event on June 14, 2014, in Rochester. Participation was offered free of charge to patients with a history of cancer, most of whom received cancer treatment at Mayo Clinic in MN, a large medical center. This annual event provides opportunity for cancer survivors to share stories with and support for each other. A booth at the event advertised the survey, and health care providers staffing 
the booth explained the study and requested oral (verbal) consent from everyone who expressed interest in participating. Persons who consented to participation received a confidential one-page survey; no identifying information was collected. Survey items included demographic characteristics, as well as the following questions:

1) How much do the following issues/symptoms bother you? [answer choices were "not at all," "a little," "somewhat," and "a lot"]: fear of recurrence, stress, fatigue, financial issues, pain, swelling, neuropathy, heart disease, difficulty sleeping, weight gain, mental fogginess, another bothersome issue.

2) Please tell us about your experience with the following integrative medicine treatments/approaches, checking all boxes that apply to you [answer choices were "previously tried or currently trying," "interested in trying," and "desires more information about this"]: exercise, improved nutrition, stress management, acupuncture, massage, dietary supplement, yoga, meditation, and wellness training.

\section{Analyses}

Descriptive statistics were used to summarize response data from this survey. A two-sided $t$ test was used to compare responses from men with those from women for each question about symptoms or concerns and IM. Similar analyses were used to compare responses from persons younger than 65 years with those from persons aged 65 years or older, as well as to compare responses from persons who received their cancer diagnosis 0 to 9 years ago with those from persons who received their diagnoses 10 or more years ago.

\section{Results}

Two hundred sixty cancer survivors attended the Cancer Survivor Day and 171 completed this survey, of whom $74 \%$ were female and $26 \%$ were male (mean [SD] age, 64.6 [12.7] years; range, 30-96 years) (Table 1). The majority of respondents $(72 \%)$ were married. The various types of cancer were lymphoma (7\%), leukemia (2\%), sarcoma (2\%), multiple myeloma ( $1 \%$ ) and breast (42\%), genitourinary (14\%), gastrointestinal (10\%), gynecologic (9\%), melanoma/skin ( $8 \%$ ), brain (4\%), head and neck (2\%), lung (2\%), and thyroid $(1 \%)$ cancer. Ten respondents reported that they were survivors of multiple types of cancer. The mean (SD) time since a cancer diagnosis was 13.1 (13.0) years.

The five symptoms or issues that respondents most commonly reported were somewhat or a lot bothersome included fear of recurrence (52\%), stress (43\%), fatigue (43\%), difficulty sleeping (33\%), and weight gain (31\%) (Fig. 1). Mental
Table 1 Characteristics of the 171 respondents

\begin{tabular}{|c|c|}
\hline Characteristic & Value $^{\mathrm{a}}$ \\
\hline Age, mean (SD), years & $64.6(12.7)$ \\
\hline \multicolumn{2}{|l|}{ Sex } \\
\hline Male & $45(26)$ \\
\hline Female & $126(74)$ \\
\hline \multicolumn{2}{|c|}{ Time since cancer diagnosis, years } \\
\hline$>20$ & $32(19)$ \\
\hline $10-20$ & $43(25)$ \\
\hline $5-9$ & $44(26)$ \\
\hline $0-4$ & $45(26)$ \\
\hline Missing data & $7(4)$ \\
\hline \multicolumn{2}{|l|}{ Cancer type } \\
\hline Breast & $71(42)$ \\
\hline Genitourinary & $24(14)$ \\
\hline Gastrointestinal & $17(10)$ \\
\hline Gynecologic & $16(9)$ \\
\hline Melanoma/skin cancer & $14(8)$ \\
\hline Lymphoma & $12(7)$ \\
\hline Brain & $6(4)$ \\
\hline Leukemia & $4(2)$ \\
\hline Head/neck & $3(2)$ \\
\hline Lung & $3(2)$ \\
\hline Sarcoma & $3(2)$ \\
\hline Multiple myeloma & $2(1)$ \\
\hline Thyroid & $2(1)$ \\
\hline \multicolumn{2}{|l|}{ Race/ethnicity } \\
\hline White & $164(96)$ \\
\hline American Indian & $2(1)$ \\
\hline Asian & $2(1)$ \\
\hline Hispanic/Latino & $2(1)$ \\
\hline With children age $<18$ years & $10(6)$ \\
\hline \multicolumn{2}{|l|}{ Marital status } \\
\hline Married & $123(72)$ \\
\hline Single & $21(12)$ \\
\hline Widowed & $20(12)$ \\
\hline Divorced & $6(4)$ \\
\hline Other & $1(1)$ \\
\hline
\end{tabular}

${ }^{a}$ Values are presented as number and percentage of patients unless specified otherwise

fogginess, pain, financial issues, neuropathy, swelling, and heart disease were reported as somewhat or a lot bothersome by fewer participants (i.e., 24, 23, 23, 17, 14, and $7 \%$, respectively). The specific "other" concerns reported by some participants (10\% total) included acid reflux, nausea, anxiety, breast neuropathy, urinary difficulties, circulatory issues, dental concerns, flaps of skin resulting from treatment and surgery, headaches, joint pain, numbness, leg pain, loneliness, neuropathy, hearing and vision problems, infections, mouth sores, and weight loss. Men were less likely than women to 
Fig 1 Survivorship concerns among the 171 respondents. Values may not total $100 \%$ if the respondent did not respond to all items

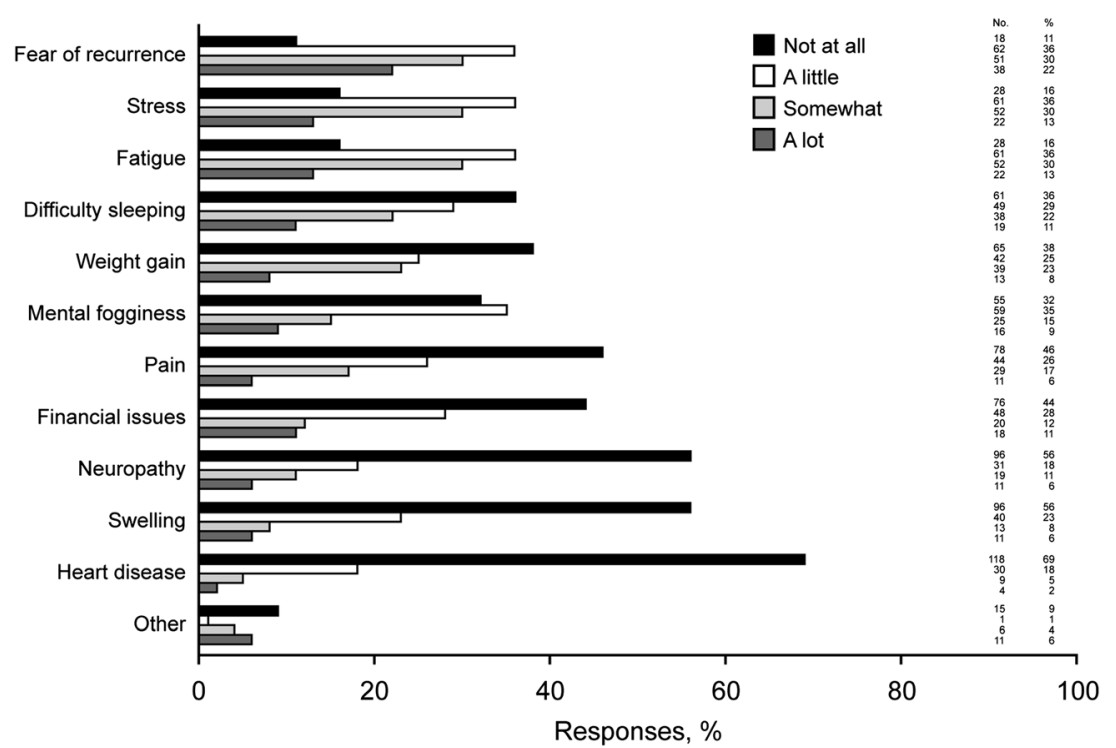

report fatigue $(P=.045)$ and weight gain $(P=.04)$. Older patients (age, $\geq 65$ years) were more likely to report heart disease but less likely to report fear of recurrence $(P=.01)$, stress $(P=.04)$, financial issues $(P=.009)$, and pain $(P=.005)$. Patients whose cancer had been diagnosed more recently $(<10$ years prior) were less likely to report heart disease $(P=.006)$.

The second question in the survey related to use of and interest in IM and other health-related resources. The resources used or tried by more than $20 \%$ of survey respondents included exercise $(75 \%)$, improved nutrition/diet (66\%), stress management (i.e., being aware of stress and how it affects health and having strategies to reduce stress) (42\%), dietary supplement (33\%), meditation (25\%), and massage (22\%) (Fig. 2). The top resources that more than $20 \%$ of survey respondents said they were interested in learning more about were dietary supplementation (29\%), acupuncture
(25\%), and wellness training (22\%). Among respondents, $18 \%$ were interested in learning more about each of the topics of massage, yoga, and meditation. Women were more likely than men to have previously tried yoga $(P=.04)$, but otherwise, no sex differences were identified in respondents' experience with, interest in, or desire for information about, the various aspects of IM. Older patients (age, $\geq 65$ years) were less experienced with nutrition and $\operatorname{diet}(P=.04)$, dietary supplementation $(P=.002)$, yoga $(P=.003)$, meditation $(P=.04)$, and wellness training $(P=.03)$. The older patients also were less interested in trying acupuncture $(P=.008)$, massage $(P=.003)$, dietary supplementation $(P=.005)$, and yoga $(P=.02)$, and fewer expressed interest in learning about nutrition and $\operatorname{diet}(P=.047)$ and acupuncture $(P=.04)$. Patients who had received a diagnosis more recently $(<10$ years prior) were more likely to have tried a dietary supplement previously or to be trying one currently $(P=.01)$.
Fig 2 Use of and interest in integrative medicine among the 171 respondents. Values may not total $100 \%$ if the respondent did not respond to all items. Other responses included tai chi; aromatherapy; prayer, spirituality, or belief in God; swimming; qigong; and water aerobics

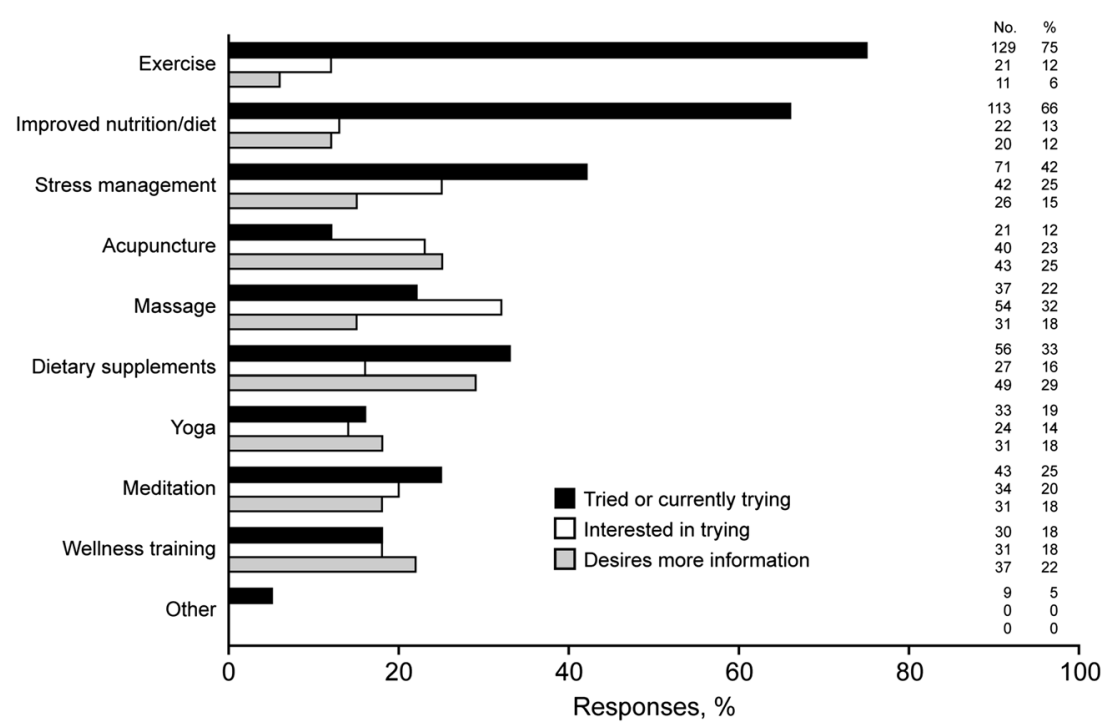




\section{Discussion}

During and after treatment, men and women with cancer are faced with the difficulty of adjusting to new physical, social, and emotional realities. Respondents to this survey-most of whom were Mayo Clinic patients_-reported substantial symptom burden, particularly related to stress, fatigue, difficulty sleeping, weight gain, and fear of recurrence. Reassuringly, most respondents had tried (or were trying currently) to exercise and eat an improved diet, both of which are important for weight control. Although many of the other IM techniques of interest to respondents are known to help mitigate symptoms and concerns (i.e., acupuncture, wellness training, massage, yoga, and meditation may all reduce stress, fatigue, sleep disturbance, and fear of recurrence), the majority of respondents had not tried most of these techniques yet. This may mean that survivors do not have enough information or training related to these techniques, or it may reflect inadequate access. Dietary supplements are often explored by cancer survivors but are often of dubious value in this clinical setting. Even so, it appears that cancer survivors are interested in knowing more about dietary supplements. These results will help guide future practice considerations.

The Mayo Clinic Comprehensive Cancer Center has a strong IM program. However, cancer survivors were not receiving all of the information they desired about many techniques, including dietary supplements, acupuncture, wellness training, yoga, meditation, stress management, nutrition and diet, and exercise. This is evident despite a Mayo Clinic class offered in Rochester for $1 \mathrm{~h}$ each week since 2012, "Moving Forward After Cancer Treatment," which promotes a healthy lifestyle, provides tips for managing difficult emotions, and discusses long-term treatment sequelae and how to be an active participant in follow-up care. Unfortunately, only a small fraction of eligible cancer survivors (averaging 100 persons annually) attend this class. By comparison, about 45,000 people nationally subscribe to a weekly electronic newsletter and the blog "Living With Cancer" (http://mayoclinic.org/ diseases-conditions/cancer/expert-blog/con-20032378), which includes information regarding survivorship care, resources, and support.

Nevertheless, this survey showed that many Mayo Clinic cancer survivors continue to be highly symptomatic and distressed. A more effective educational program and coordinated inclusion of these IM techniques in cancer survivorship care may help reduce survivors' emotional and physical symptoms. These educational programs would ideally be accessible in multiple ways, including in person and online, and would offer practical tips for application and demonstration of techniques. Follow-up discussion or coaching may be helpful, as may regular discussions with a health care provider regarding what strategies are being used for managing emotional and physical symptoms.
Interestingly, sex differences in the survey responses were minimal and may have been spurious, given the small sample size and lack of correction for multiple testing. Likewise, time since diagnosis did not seem to impact symptom burden (except for heart disease, which likely is confounded by older age in those persons who received their cancer diagnosis more than 10 years ago), or to affect IM use and interest (except for experience with nutritional supplements, which appeared more common in persons whose cancer was diagnosed recently but which also may reflect age-related trends). Older age ( $\geq 65$ years) was consistently associated with fewer symptoms and issues - except for heart disease, which increases with age in all populations - and older survivors also reported less experience with, interest in trying, and interest in learning about various IM techniques. This finding supports prior findings that cancer diagnosis and treatment are often particularly distressing to younger patients, and suggests that future IMbased interventions may be most appealing to younger cancer survivors. Importantly, this study surveyed a convenience sample of survivors who self-selected to participate, so the generalizability of these results is uncertain.

\section{Conclusion}

Cancer survivors, and particularly those survivors younger than 65 years, continue to have treatment adverse effects many years into the survivorship period. They use various IM-related techniques to help manage their symptoms, and they have a strong interest in learning more about dietary supplements, acupuncture, wellness training, massage, yoga, and meditation. It is critical that oncology providers help cancer survivors cope with long-term consequences of their diagnoses and treatments. Education about and access to IM may help cancer survivors optimize multiple aspects of their health and should be included in comprehensive cancer survivorship programs.

Acknowledgments The authors gratefully acknowledge the support of the John P. and Carole E. Gregory Foundation, which helped make this work possible. This research was also made possible by CTSA Grant Number KL2 TR000136-09 and UL1 TR000135 from the National Center for Advancing Translational Science (NCATS), a component of the National Institutes of Health (NIH). Its contents are solely the responsibility of the authors and do not necessarily represent the official views of the National Institutes of Health.

Conflict of interest The authors have no conflicts of interest to report. The authors have full control of all primary data and agree to allow the journal to review their data if requested.

\section{References}

1. Glare PA, Davies PS, Finlay E, Gulati A, Lemanne D, Moryl N et al (2014) Pain in cancer survivors. J Clin Oncol 32(16):1739-1747 
2. Pachman DR, Barton DL, Swetz KM, Loprinzi CL (2012) Troublesome symptoms in cancer survivors: fatigue, insomnia, neuropathy, and pain. J Clin Oncol 30(30):3687-3696

3. Bower JE, Bak K, Berger A, Breitbart W, Escalante CP, Ganz PA et al (2014) Screening, assessment, and management of fatigue in adult survivors of cancer: an American Society of Clinical Oncology clinical practice guideline adaptation. J Clin Oncol 32(17):1840-1850

4. Hershman DL, Lacchetti C, Dworkin RH, Lavoie Smith EM, Bleeker J, Cavaletti G, American Society of Clinical Oncology et al (2014) Prevention and management of chemotherapy-induced peripheral neuropathy in survivors of adult cancers: American Society of Clinical Oncology clinical practice guideline. J Clin Oncol 32(18): 1941-1967

5. Paskett ED, Dean JA, Oliveri JM, Harrop JP (2012) Cancer-related lymphedema risk factors, diagnosis, treatment, and impact: a review. J Clin Oncol 30(30):3726-3733

6. Lenihan DJ, Cardinale DM (2012) Late cardiac effects of cancer treatment. J Clin Oncol 30(30):3657-3664, Erratum in: J Clin Oncol. 2012 Dec 20;30(36):4590

7. Ness S, Kokal J, Fee-Schroeder K, Novotny P, Satele D, Barton D (2013) Concerns across the survivorship trajectory: results from a survey of cancer survivors. Oncol Nurs Forum 40(1):35-42

8. Lynch BM, Dunstan DW, Vallance JK, Owen N (2013) Don't take cancer sitting down: a new survivorship research agenda. Cancer 119(11):1928-1935

9. Zheng Y, Luo J, Bao P, Cai H, Hong Z, Ding D et al (2014) Longterm cognitive function change among breast cancer survivors. Breast Cancer Res Treat 146(3):599-609

10. Denlinger CS, Ligibel JA, Are M, Baker KS, Demark-Wahnefried W, Friedman DL et al (2014) Survivorship: cognitive function, version 1.2014. J Natl Compr Cancer Netw 12(7):976-986

11. Ferguson RJ, Ahles TA, Saykin AJ, McDonald BC, Furstenberg CT, Cole BF et al (2007) Cognitive-behavioral management of chemotherapy-related cognitive change. Psychooncology 16(8): 772-777

12. Raggio GA, Butryn ML, Arigo D, Mikorski R, Palmer SC (2014) Prevalence and correlates of sexual morbidity in long-term breast cancer survivors. Psychol Health 29(6):632-650

13. Ganz PA, Rowland JH, Desmond K, Meyerowitz BE, Wyatt GE (1998) Life after breast cancer: understanding women's healthrelated quality of life and sexual functioning. J Clin Oncol 16(2): 501-514

14. Carter N, Bryant-Lukosius D, DiCenso A, Blythe J, Neville AJ (2011) The supportive care needs of men with advanced prostate cancer. Oncol Nurs Forum 38(2):189-198

15. Lebel S, Maheu C, Lefebvre M, Secord S, Courbasson C, Singh M et al (2014) Addressing fear of cancer recurrence among women with cancer: a feasibility and preliminary outcome study. J Cancer Surviv 8(3):485-496
16. Eisenberg SA, Kurita K, Taylor-Ford M, Agus DB, Gross ME, Meyerowitz BE (2015) Intolerance of uncertainty, cognitive complaints, and cancer-related distress in prostate cancer survivors. Psychooncology 24(2):228-235

17. McCarthy M (2014) Cancer survivors face substantial financial burdens, US report says. BMJ 348:g4017

18. Feng Y, Wang XY, Li SD, Zhang Y, Wang HM, Li M et al (2011) Clinical research of acupuncture on malignant tumor patients for improving depression and sleep quality. J Tradit Chin Med 31(3): 199-202

19. Mehling WE, Jacobs B, Acree M, Wilson L, Bostrom A, West J et al (2007) Symptom management with massage and acupuncture in postoperative cancer patients: a randomized controlled trial. J Pain Symptom Manag 33(3):258-266

20. Molassiotis A, Sylt P, Diggins H (2007) The management of cancerrelated fatigue after chemotherapy with acupuncture and acupressure: a randomised controlled trial. Complement Ther Med 15(4):228-237

21. Walker EM, Rodriguez AI, Kohn B, Ball RM, Pegg J, Pocock JR et al (2010) Acupuncture versus venlafaxine for the management of vasomotor symptoms in patients with hormone receptor-positive breast cancer: a randomized controlled trial. J Clin Oncol 28(4):634-640

22. Molassiotis A, Bardy J, Finnegan-John J, Mackereth P, Ryder DW, Filshie $\mathrm{J}$ et al (2012) Acupuncture for cancer-related fatigue in patients with breast cancer: a pragmatic randomized controlled trial. J Clin Oncol 30(36):4470-4476

23. Balk J, Day R, Rosenzweig M, Beriwal S (2009) Pilot, randomized, modified, double-blind, placebo-controlled trial of acupuncture for cancer-related fatigue. J Soc Integr Oncol 7(1):4-11

24. Hervik J, Mjaland O (2010) Quality of life of breast cancer patients medicated with anti-estrogens, 2 years after acupuncture treatment: a qualitative study. Int J Womens Health 2:319-325

25. Loprinzi CE, Prasad K, Schroeder DR, Sood A (2011) Stress Management and Resilience Training (SMART) program to decrease stress and enhance resilience among breast cancer survivors: a pilot randomized clinical trial. Clin Breast Cancer 11(6):364-368

26. Lengacher CA, Reich RR, Paterson CL, Jim HS, Ramesar S, Alinat CB, et al (2014) The effects of mindfulness-based stress reduction on objective and subjective sleep parameters in women with breast cancer: a randomized controlled trial. Psychooncology. doi:10.1002/pon. 3603

27. Johns SA, Brown LF, Beck-Coon K, Monahan PO, Tong Y, Kroenke K (2014) Randomized controlled pilot study of mindfulness-based stress reduction for persistently fatigued cancer survivors. Psychooncology. doi:10.1002/pon.3648

28. Garland SN, Carlson LE, Stephens AJ, Antle MC, Samuels C, Campbell TS (2014) Mindfulness-based stress reduction compared with cognitive behavioral therapy for the treatment of insomnia comorbid with cancer: a randomized, partially blinded, noninferiority trial. J Clin Oncol 32(5):449-457 